\title{
AN INVARIANT FOR CONTINUOUS FACTORS OF MARKOV SHIFTS
}

\author{
BRUCE KITCHENS
}

\begin{abstract}
Let $\Sigma_{A}$ and $\Sigma_{B}$ be subshifts of finite type with Markov measures $(p, P)$ and $(q, Q)$. It is shown that if there is a continuous onto measure-preserving factor map from $\Sigma_{A}$ to $\Sigma_{B}$, then the block of the Jordan form of $Q$ with nonzero eigenvalues is a principal submatrix of the Jordan form of $P$. If $\Sigma_{A}$ and $\Sigma_{B}$ are irreducible with the same topological entropy, then the same relationship holds for the matrices $A$ and $B$. As a consequence, $\zeta_{B}(t) / \zeta_{A}(t)$, the ratio of the zeta functions, is a polynomial. From this it is possible to construct a pair of equalentropy subshifts of finite type that have no common equal-entropy continuous factor of finite type, and a strictly sofic system that cannot have an equal-entropy subshift of finite type as a continuous factor.
\end{abstract}

1. Introduction. Let $A$ be an $l \times l$ matrix of 0 's and 1's. The subshift of finite type, $\Sigma_{A}$, determined by $A$ is the closed invariant subspace of $\{1, \ldots, l\}^{\mathbf{Z}}$ consisting of all $x=\ldots x_{-1} x_{0} x_{1} \ldots$ such that $A_{x_{i} x_{i+1}}=1$ for all $i$, together with the shift transformation [9]. A Markov measure is defined on this space by a pair $(p, P)$, where $P$ is a stochastic matrix compatible with $A$ (i.e., positive, row-sum 1 , and $P_{i j}>0$ exactly when $\left.A_{i j}=1\right)$ and $p$ is a probability vector with $p P=p$ [9].

The following dynamical properties of subshifts of finite type will be used. A subshift of finite type is topologically transitive when its transition matrix is irreducible [9]. The irreducibility of the transition matrix is also the condition needed for erogodicity with respect to any Markov measure [9]. The zeta function of a dynamical system is $\zeta(t)=\exp \left[\sum_{n=1}^{\infty}\left(N_{n}\right) t_{n} / n\right]$, where $N_{n}$ is the number of points fixed by the $n$th power of the transformation. For a subshift of finite type, $\Sigma_{A}$, this has the form $\zeta_{A}(t)=\left[t^{l} C_{A}(1 / t)\right]^{-1}$, where $C_{A}(x)$ is the characteristic polynomial of $A$ [2]. The topological entropy of an irreducible subshift of finite type is $\log \lambda$, where $\lambda$ is the largest positive real eigenvalue [9], and the measuretheoretic entropy with respect to a $\operatorname{Markov}$ measure $(p, P)$ is $-\Sigma_{i j} p_{i} P_{i j} \log P_{i j}[9]$. An irreducible subshift of finite type has a unique measure of maximal entropy [9], which is a Markov measure whose matrix has the form $P=\frac{1}{\lambda} R^{-1} A R$ ( $R$ a diagonal matrix with strictly positive diagonal entries). Any continuous onto finite-to-one factor map between two subshifts of finite type will carry the measure of maximal entropy of one to the measure of maximal entropy of the other [3]. The CurtisHedlund-Lyndon theorem [6] asserts that any continuous factor map between subshifts of finite type is a block map composed with some power of the shift. This

Received by the editors March 10, 1981.

1980 Mathematics Subject Classification. Primary 58F20.

Key words and phrases. Subshift of finite type, Markov shift, zeta function, factor maps.

(C) 1981 American Mathematical Society 0002-9939/81/0000-0581/\$02.00 
means that by going to a higher block presentation of the domain shift, any continuous factor map can be expressed as a one-block map [1].

\section{2. $P$ 's and $Q$ 's.}

THEOREM. If $\Sigma_{A}$ and $\Sigma_{B}$ are subshifts of finite type and if there is a continuous onto factor map between them that takes $(p, P)$ to $(q, Q)$, then the block of the Jordan form of $Q$ with nonzero eigenvalues is a principal submatrix of the Jordan form of $P$.

Proof. We may assume that the factor map $\phi: \Sigma_{A} \rightarrow \Sigma_{B}$ is a one-block map [10]. Take $L_{A}$ to be the alphabet of $\Sigma_{A}$ and $L_{B}$ the alphabet of $\Sigma_{B} . R$ will be the relation matrix that represents the equivalence relation defined on $L_{A}$ by $\phi$ : for $i \in L_{A}$, $a \in L_{B}$

$$
R_{i a}= \begin{cases}1 & \text { if } \phi(i)=a, \\ 0 & \text { otherwise. }\end{cases}
$$

A time-zero cylinder set is defined by $\left[a_{1}, \ldots, a_{n}\right]=\left\{x: x_{0}=a_{1}, \ldots, x_{n-1}=a_{n}\right\}$. The inverse image of a time-zero cylinder set in $\Sigma_{B}$ is a finite union of time-zero cylinder sets in $\Sigma_{A}$, of the same length. For any $\left[a_{1}, \ldots, a_{n}\right] \subseteq \Sigma_{B}$ define $u^{\left[a_{1}, \ldots, a_{n}\right]}$ $\in \mathbf{R}^{\left|L_{A}\right|}$ and $v^{\left[a_{1}, \ldots, a_{n}\right]} \in \mathbf{R}^{\left|L_{B}\right|}$ by $\left(u^{\left[a_{1}, \ldots, a_{n}\right]}\right)_{i}=\sum p_{i_{1}} P_{i_{1} i_{2}} \cdots P_{i_{n-1} i_{n}}$, where the sum is taken over all $\left[i_{1}, \ldots, i_{n}\right]$ in $\phi^{-1}\left[a_{1}, \ldots, a_{n}\right]$ such that $i_{n}=i$,

$$
\left(v^{\left[a_{1}, \ldots, a_{n}\right]}\right)_{a}= \begin{cases}q_{a_{1}} Q_{a_{1} a_{2}} \cdots Q_{a_{n-1} a_{n}} & \text { if } a_{n}=a, \\ 0 & \text { otherwise. }\end{cases}
$$

Let $U$ be the collection of all $u^{\left[a_{1}, \ldots, a_{n}\right]}$ and $V$ that of all $v^{\left[a_{1}, \ldots, a_{n}\right]} . V$ generates all of $\mathbf{R}^{\left|L_{B}\right|}$; let $\mathscr{U}$ be the subspace of $\mathbf{R}^{\left|L_{A}\right|}$ generated by $U$. A computation using the measure-preserving property of $\phi$ shows the diagram

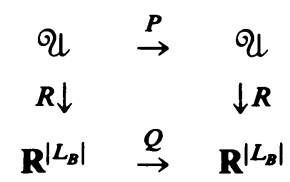

commutes, where the matrices operate by left multiplication, i.e. $x P R=x R Q$ for all $x \in \mathcal{Q}$. Since $R$ has rank $\left|L_{B}\right|$, we have the desired result. Notice that this linear algebra situation is equivalent to the existence of such a factor map.

Corollary A. If $\Sigma_{A}, \Sigma_{B}$ are irreducible subshifts of finite type and $\Sigma_{B}$ is a continuous finite-to-one factor of $\Sigma_{A}$, then the block of the Jordan form of $B$ with nonzero eigenvalues is a principal submatrix of the Jordan form of $A$. In particular, if $\zeta_{A}(t)$ and $\zeta_{B}(t)$ are the zeta functions, then $\zeta_{B}(t) / \zeta_{A}(t)$ is a polynomial.

Proof. $\Sigma_{A}$ and $\Sigma_{B}$ have the same topological entropy, $\log \lambda$. The matrices $P, Q$ for the measures of maximal entropy are $P=\frac{1}{\lambda} R^{-1} A R$ and $Q=\frac{1}{\lambda} S^{-1} B S$, where $R$, $S$ are the appropriate diagonal matrices of full rank. The desired result is obtained by applying the thoerem. Recalling that $\zeta_{A}(t)=\left(t^{\left|L_{A}\right|} C_{A}(1 / t)\right)^{-1}$, we have the observation about the ratio of the zeta functions. We also have a completely topological proof of this corollary, and M. Nasu [8] has proved the fact about the ratio of the zeta functions using graph-theoretic techniques. 
COROllary B. There exist equal-entropy mixing [1] subshifts of finite type that have no common equal-entropy continuous factor. This is in contrast to the AdlerMarcus Theorem, which asserts that any such shifts have a common equal-entropy continuous extension [1].

Proof. Take

$$
A=\left[\begin{array}{l}
001 \\
101 \\
010
\end{array}\right], \quad B=\left[\begin{array}{l}
00001 \\
10000 \\
01000 \\
00100 \\
00011
\end{array}\right],
$$

then $C_{A}(x)=x^{3}-x-1$, which is irreducible, and $C_{B}(x)=x^{5}-x^{4}-1=$ $\left(x^{3}-x-1\right)\left(x^{2}-x+1\right)$. These are both mixing and have the same entropy. Since $C_{A}(x)$ is irreducible over $\mathbf{Z}$, any continuous finite-to-one factor of $\Sigma_{A}$ must have the same zeta function. $\Sigma_{B}$ has a fixed point, so any factor of it must have a fixed point. There is no subshift of finite type that meets both of these requirements.

COROllary C. There exists a mixing strictly sofic system [11] that has no equal-entropy subshift of finite type as a continuous factor. This should be compared to the fact that any sofic system is a continuous equal-entropy factor of a subshift of finite type [4].

Proof. Begin with $\Sigma_{A}$ as in the previous corollary. Obtain a strictly sofic system of the same entropy by identifying the pair of two-blocks [2, 3] and [3, 2]. This sofic system has a fixed point. Any subshift of finite type that is an equal-entropy continuous factor of this system is also one for $\Sigma_{A}$. We already know there is no such shift. This construction was noticed by Brian Marcus.

COROllary D. Any equal-entropy continuous factor of the full shift which is a subshift of finite type is shift equivalent (in the sense of Williams [12]) to the same full shift. This was previously proved in [7].

Proof. Any equal-entropy continuous factor of the full $n$-shift that is a subshift of finite type has the zeta function $(1-n t)^{-1}$. R. Williams [12] has shown that any subshift of finite type with this zeta function is shift equivalent to the full $n$-shift. It is possible to deduce Corollaries B and C from the work of J. Cuntz and W. Krieger [5].

I would like to thank my advisor, Brian Marcus, for getting me started and keeping me going on these questions.

\section{REFERENCES}

1. R. Adler and B. Marcus, Topological entropy and equivalences of dynamical systems, Mem. Amer. Math. Soc., no. 219, 1979.

2. R. Bowen and O. Lanford, Zeta functions of restrictions of the shift transformation, Proc. Sympos. Pure Math., vol. 14, Amer. Math. Soc., Providence, R.I., 1970.

3. E. Coven and M. Paul, Endomorphisms of irreducible shifts of finite type, Math. Systems Theory 8 (1974), 167-175. 
4. __ Sofic systems, Israel J. Math. 20 (1975), 165-177.

5. J. Cuntz and W. Krieger, Topological Markov chains with dicycle dimension groups (preprint).

6. G. A. Hedlund, Endomorphisms and automorphisms of the shift dynamical system, Math. Systems Theory 3 (1969), 320-375.

7. B. Marcus, Factors and extensions of full shifts, Monatsh. Math. 88 (1979), 239-247.

8. M. Nasu, Uniformly finite-to-one and onto extensions of homomorphisms between strongly connected graphs, preprint, Research Institute of Electrical Communication, Tôhoku University, Sendai, Japan.

9. W. Parry, Intrinsic Markov chains, Trans. Amer. Math. Soc. 112 (1964), 55-66.

10. W. Parry and R. F. Williams, Block coding and a zeta function for finite Markov chains (preprint).

11. B. Weiss, Subshifts of finite type and sofic systems, Monatsh. Math. 77 (1973), 462-474.

12. R. F. Williams, Classification of subshifts of finite type, Ann. of Math. 98 (1973), 120-153; erratum 99 (1974), 380-381.

Department of Mathematics, University of North Carolina, Chapel Hill, North Carolina 27514

Current address: Department K64, IBM Research Center, San Jose, California 95193 